\title{
Experimental Combustion Dynamics Behavior Of A Multi- Element Lean Direct Injection (LDI) Gas Turbine Combustor
}

\author{
Waldo A. Acosta ${ }^{1}$ \\ Army Research Laboratory, Cleveland, $\mathrm{OH}$ 44135, USA \\ Clarence T. Chang ${ }^{2}$ \\ NASA Glenn Research Center, Cleveland, OH 44135, USA
}

\begin{abstract}
An experimental investigation of the combustion dynamic characteristics of a research multi-element lean direct injection (LDI) combustor under simulated gas turbine conditions was conducted. The objective was to gain a better understanding of the physical phenomena inside a pressurized flametube combustion chamber under acoustically isolated conditions. A nine-point swirl venturi lean direct injection (SV-LDI) geometry was evaluated at inlet pressures up to $2,413 \mathrm{kPa}$ and non-vitiated air temperatures up to $867 \mathrm{~K}$. The equivalence ratio was varied to obtain adiabatic flame temperatures between $1388 \mathrm{~K}$ and $1905 \mathrm{~K}$. Dynamic pressure measurements were taken upstream of the SV-LDI, in the combustion zone and downstream of the exit nozzle. The measurements showed that combustion dynamics were fairly small when the fuel was distributed uniformly and mostly due to fluid dynamics effects. Dynamic pressure fluctuations larger than $40 \mathrm{kPa}$ at low frequencies were measured at $653 \mathrm{~K}$ inlet temperature and $1117 \mathrm{kPa}$ inlet pressure when fuel was shifted and the pilot fuel injector equivalence ratio was increased to 0.72 .
\end{abstract}

\section{Nomenclature}
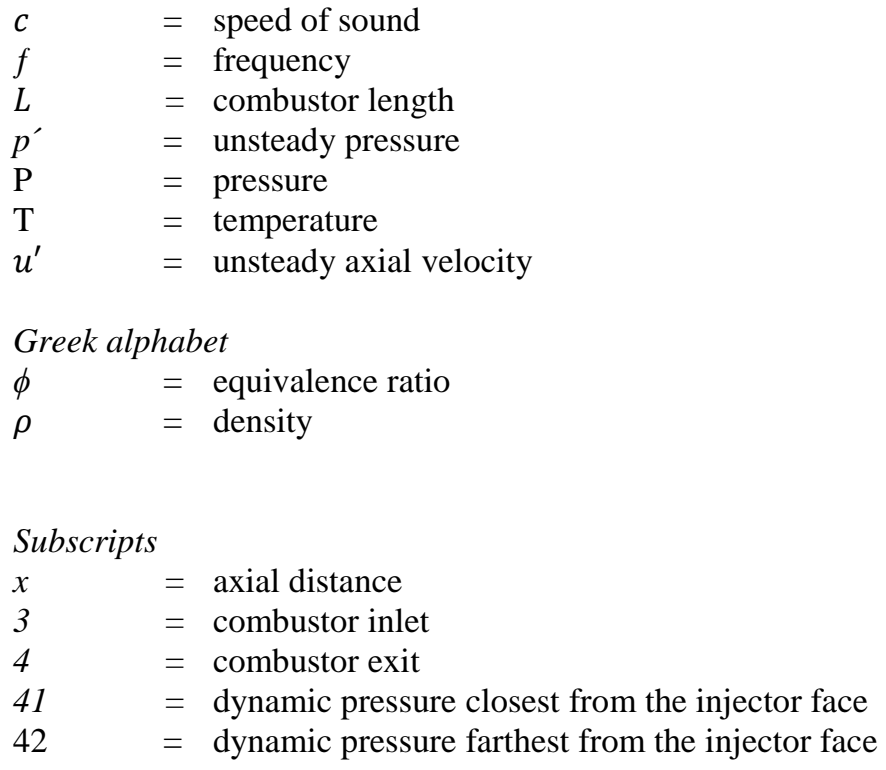

Acronyms

$C R Z=$ central recirculation zone

${ }^{1}$ Aerospace Engineer, Vehicle Technology Directorate, 21000 Brookpark Rd./MS 5-10, AIAA Senior Member

${ }^{2}$ Aerospace Engineer, Combustion Branch, 21000 Brookpark Rd./Mail Stop 5-10, Cleveland, OH. 
$L D I \quad=$ lean direct injection

$\mathrm{PVC}=$ precessing vortex core

$S V-L D I=$ swirl venturi lean direct injection

$\mathrm{VBB}=$ vortex breakdown bubble

\section{Introduction}

$\mathrm{T}$ HE Lean Direct Injection (LDI) combustor concept is potentially the only useful lean-burn mode for ultra high pressure combustion above $6,000 \mathrm{kPa}$. At the elevated combustor inlet temperature and pressure, the fuel ignition delay time is almost non-existent and the fuel burns while it mixes, hence the reference to "direct" injection of the fuel into the combustion zone. Multiple small diameter fuel nozzles are used to reduce the transport distance and speed up mixing as well as improving fuel-air mixture homogeneity to reduce hot spots. One simple injector element design used at NASA GRC uses a helical axial swirler that feed into a convergent-divergent venturi where the swirling shear gradient is accentuated to enhance the liquid fuel break up process ${ }^{1}$.

Swirling flows are the most common method used in gas turbine engines to stabilize the flame and promote fuel air mixing. The swirling flow creates a central recirculation zone (CRZ) that anchors the flame and provides stability. In addition, this CRZ is also associated with a precessing vortex core (PVC) where the axis of rotation of the swirling flow rotates around the geometric center of the flow field ${ }^{2}$. Lean-burning swirl stabilized flames have exhibited combustion dynamics that are associated with swirl-acoustic interactions. It has been reported that there is a non-linear interaction between the PVC and the thermo-acoustic modes in the flames of premixed methane-air combustors. The majority of the work on the topic of combustion instabilities in swirl-stabilized flames has been premixed gaseous flows at atmospheric conditions as reported by $\mathrm{Candel}^{3}$.

Liquid fuels introduce another dimension with its spray droplets and the transport of fuel and air to the flame creating changes in the flame structure due to the non-uniformity in the mixture. The swirling flame characteristics can be altered by the uneven response of the fuel and air to acoustic waves. There have been some studies utilizing liquid fuels conducted with partially pre-mixed and non-premixed flames at atmospheric and moderate pressures $(1034 \mathrm{kPa})$ and inlet temperatures up to $800 \mathrm{~K}^{4-10}$.

A piloted lean direct injector was studied by DeLaat et $\mathrm{al}^{4}$. They found that in general the amplitude of the instability increases as the equivalence ratio increases. Their simulation confirmed the experimental observations. The flame spectra and the combustion instability in a turbulent liquid-fueled swirl-stabilized single LDI combustor were studied by Yi et al. ${ }^{5,6}$. They found the flame spectra and the dynamics response to be very similar to that of lean premixed gaseous combustion. Another liquid-fueled swirl-stabilized single LDI combustor was studied by Huang et al. ${ }^{7}$. They utilized dynamic mode decomposition (DMD) analysis to identify the coupling between axial acoustics and the vortex breakdown bubble in the lower frequency region. In addition, DMD analysis also indicated the presence of another hydrodynamic mode, the PVC. The possibility of nonlinear coupling between acoustics and PVC modes was also identified. Some studies of low frequency combustion instabilities of a gas turbine combustor flame tube were also conducted ${ }^{8,9}$. Strong, low frequency combustion instability was generated when they operated the combustor at off-design conditions near the idle condition. The strong combustion instabilities present were attributed to a lean-limit instability ${ }^{8}$ and an "equivalence ratio oscillation"" coupled to a Helmholtz mode.

All the previously cited work was for a single injector, some of them specifically design to generate combustion instabilities in laboratory settings. Multiple injector systems may have combustion dynamic characteristics different from single injectors due to injector-to-injector interactions. An assessment of combustion dynamics in a low- $\mathrm{NO}_{\mathrm{x}}$ second-generation swirl-venturi lean direct injection combustion concept was conducted by Tacina et al. ${ }^{9}$. The injector consisted of a pilot injector surrounded by twelve swirl-venturi injectors. A fuel staging study was conducted at low inlet temperature and pressure $(505 \mathrm{~K}, 1,690 \mathrm{kPa})$ utilizing only the pilot, four of the airblast atomizers, and four of the simplex atomizers. Shifting the fuel flow from the simplex to the airblast atomizers reduced the magnitude of the combustion instability near the $650 \mathrm{~Hz}$ frequency considerably. Their conclusion was that the peak dynamic was caused by airflow because it was still present when no fuel was flowing, but was amplified by combustion. Also that the complex frequency spectrum present at lower frequencies, $200-400 \mathrm{~Hz}$, was consistent with an airblast fuel-air mixer due to the interaction between shear layers and multiple PVC's from the multiple air swirlers.

Szedlmayer ${ }^{10}$ compared the flame response of a multi-nozzle combustor to a single-nozzle system under atmospheric pressure conditions using the same nozzle design. He found that the flame structure varied significantly between the two cases. In the multi-nozzle flame the highest heat release rate occurred in the interaction region between flames. In the single-nozzle system the highest heat release rate occurred near where the flame impinges on the combustor wall. 
This work aims to characterize the combustion dynamics behavior of liquid-fueled, multi-element, swirl stabilized LDI combustors at higher inlet pressures $(2,413 \mathrm{kPa})$ and temperatures $(867 \mathrm{~K})$ conditions.

\section{Experimental Facility}

The experiments were conducted in stand 2 of the CE-5B flametube combustion test rig at the Glenn Research Center. The experimental facility can supply non-vitiated air preheated to $867 \mathrm{~K}$ at pressures up to $3,103 \mathrm{kPa}$ with flow rates up to $4.54 \mathrm{~kg} / \mathrm{s}$. The flametube, shown in Fig. 1, had a nominal $76.2 \mathrm{~mm}$ x $76.2 \mathrm{~mm}$ square cross section and was lined with an aluminum oxide ceramic capable of withstanding temperatures up to $2033 \mathrm{~K}$. The flametube test section was acoustically isolated by choking the flow at the inlet with a venturi located just upstream of the fuel injector and a nozzle downstream of the flametube to choke the exhaust gases. It should be noted that it was not possible to achieve choked flow at both the inlet and exit of the flametube for all the experimental conditions due to facility limitations.

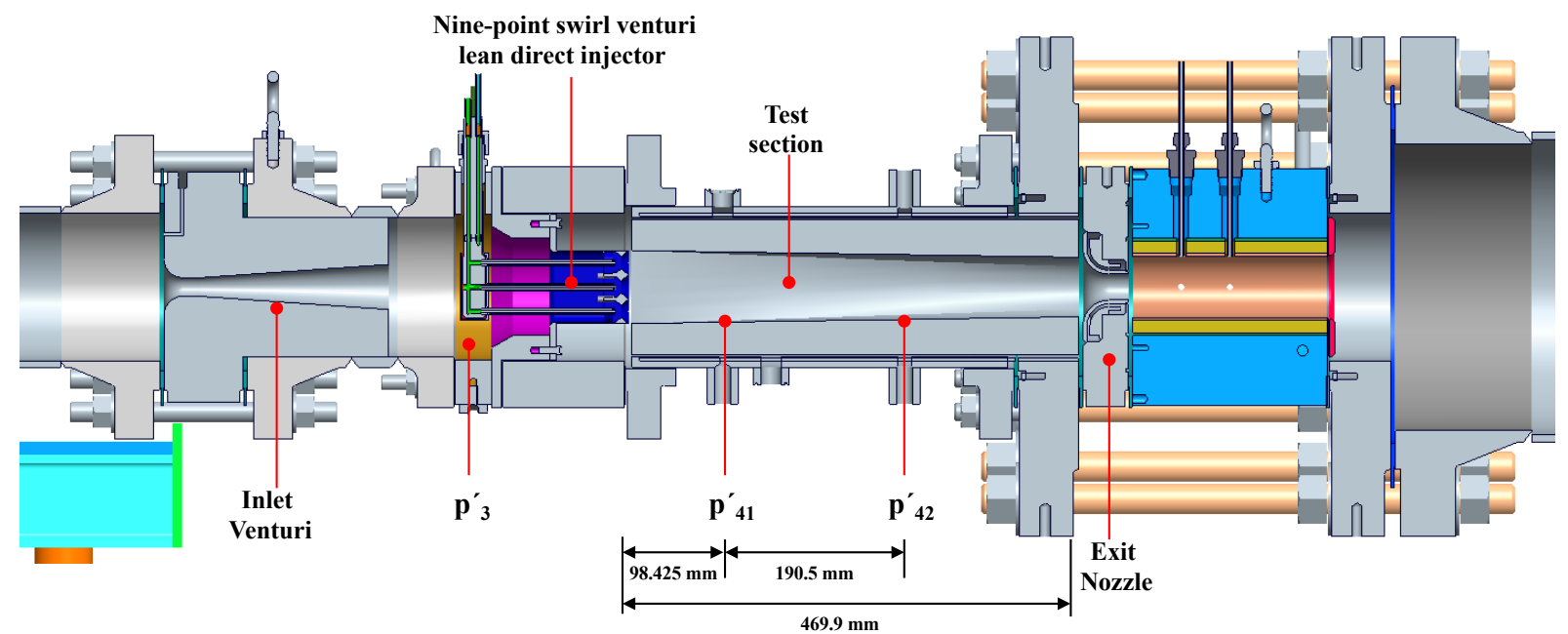

Figure 1. Schematic of the test facility showing the locations of the dynamic pressure measurements.

A nine-point swirl venturi lean direct injection (SV-LDI) geometry, as described in Ref. 1 and shown in Fig. 2, was used in these experiments. It consists of nine fuel/air mixers arranged in a $3 \times 3$ array spaced $25.4 \mathrm{~mm}$ apart measured center-to-center. Each of the fuel/air mixers has a simplex fuel injector downstream of a swirler consisting of a set of six helical blades angled at $60^{\circ}$. Each blade has an inside diameter of $9.4 \mathrm{~mm}$ and an outside diameter of $22 \mathrm{~mm}$. All the swirl blades rotated in the same direction, counterclockwise aft looking forward. Based on Beer and Chigier's ${ }^{12}$ definition, the calculated swirl number is 1.02 . The air swirler array has a measured effective area of $1000 \mathrm{~mm}^{2}$. The fuel injector tip is located at the throat of the fuel/air mixer venturi, which has a $12.7 \mathrm{~mm}$ diameter. The flow number FNUS ${ }^{9}$ of each simplex fuel injector is 2.9. The SV-LDI injector used in this experiment had three separate fuel circuits or manifolds. One circuit fuels the nozzles at the corners (number 1, 3, 7, and 9), the second one fuels the nozzles in between the corners (number 2, 4, 6, and 8), and the third one fuels the center nozzle (number 5), which will be considered the primary or pilot nozzle. Each fuel circuit was controlled independently, which allowed the fuel to be staged.

\section{Test Conditions and Data Acquisition}

The dynamic characteristics of the SV-LDI injector were measured over a range of combustor operating conditions. The nominal range of conditions is shown in Table 1.

Steady state data was acquired at a rate of $1 \mathrm{~Hz}$ utilizing the NASA Glenn ESCORT data acquisition system. It recorded facility conditions such as temperature, pressures, and flow rates.

Table 1. Range of nominal combustor operating conditions

Inlet pressure $(\mathrm{kPa}) \ldots \ldots \ldots \ldots \ldots \ldots \ldots \ldots \ldots, 1,034$ to 2,413

Inlet temperature $(\mathrm{K})$ 653 to 828

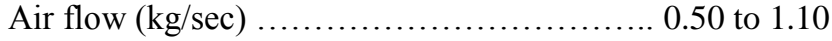

Equivalence ratio ........................... 0.29 to 0.52 

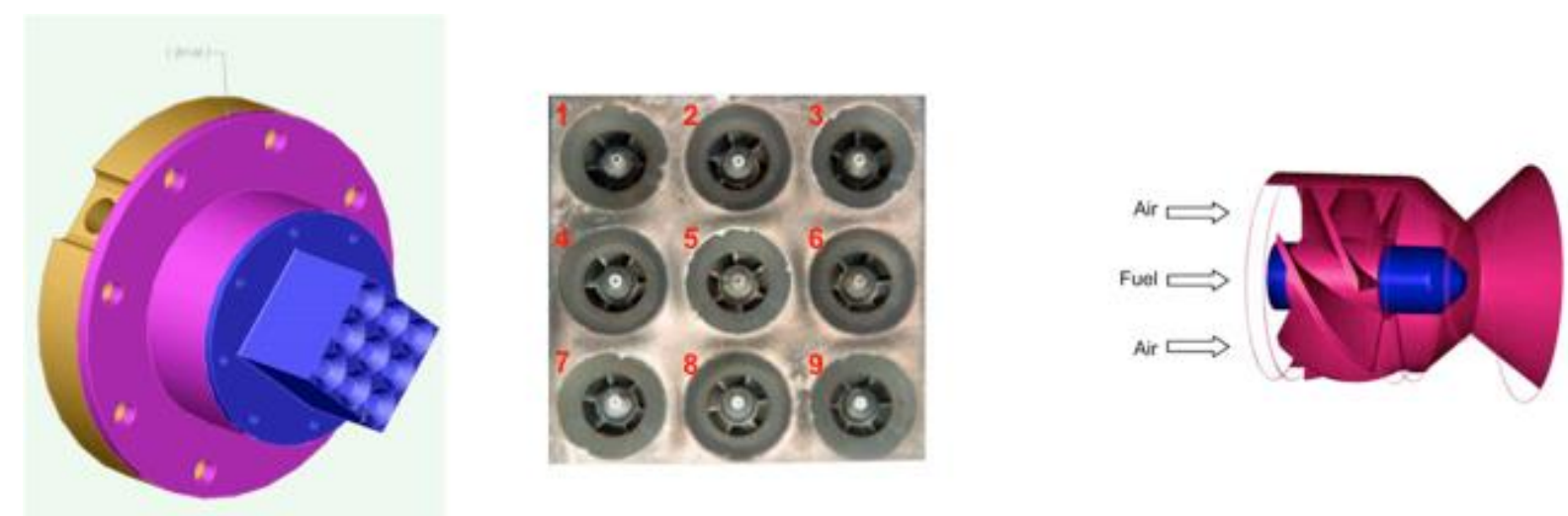

Figure 2. Schematic of the SV-LDI injector, on the left is an isometric view of the injector, end view in the center, and the relative spatial positioning of the air swirler, fuel nozzle, and venturi for each injector.

Dynamic pressure fluctuations were recorded using a Data Translation DT9841-sb high-speed data acquisition system. Dynamic data was typically recorded at $30 \mathrm{kHz}$ for a 30 second period. The dynamic pressures were measured upstream $\left(\mathrm{p}_{3}^{\prime}\right)$ and downstream of the SV-LDI $\left(\mathrm{p}^{\prime}{ }_{41}\right.$ and $\left.\mathrm{p}^{\prime}{ }_{42}\right)$. The measurements were made with small piezoelectric transducers, model 112A22 procured from PCB Piezotronics, Inc. The dynamic pressure measurements were made using nitrogen-cooled semi-infinite probes as discussed by Englund ${ }^{13}$ and Samuelson ${ }^{14}$. Each probe consists of a $30.175 \mathrm{~m}$ coil of $6.35 \mathrm{~mm}$ outer diameter steel tubing. The probe tip consisted of $6.35 \mathrm{~mm}$ outer diameter steel tubing, $920 \mathrm{~mm}$ long, that isolated the transducer from the hot gases and thermal radiation effects. The probe's tip was mounted flushed with the flametube wall. The lengths of the tip probe and the coil tubing were kept the same for each probe to minimize channel-to-channel variations. The $30.175 \mathrm{~m}$ coil is there to attenuate the acoustic waves in a way that the amplitude of any reflected wave that returns to the transducer's face is well below the amplitude of the direct wave.

The coil and the probe tip tubing were constantly purged with nitrogen to prevent the hot air and combustion gases from entering the probe and damaging the transducer. An automatic valve was used to maintain the pressure inside the tubing higher than the flametube pressure. The pressure upstream of the SV-LDI injector was used as the reference pressure since it is only a few psi higher than the mean pressure inside the flametube. The valve was set up to supply an outlet pressure approximately $69 \mathrm{kPa}$ above the reference pressure.

The flametube was instrumented with thermocouples to measure inlet and exhaust temperatures. Pressure transducers were used to measure inlet and exit pressures as well as pressure drops across the inlet venture, SV-LDI injector, flametube, and exit nozzle. A calibrated venturi was used to measure air mass flow.

\section{Results and Discussion}

\section{A. Combustion Dynamics}

The combustion dynamic characteristics of a SV-LDI fuel injector were measured over a range of inlet temperatures, pressures, and equivalence ratios as previously shown in Table 1. Figure 3 shows the peak-to-peak pressure fluctuations inside the flame tube $98 \mathrm{~mm}\left(p^{\prime}{ }_{41}\right)$ downstream from the face of the SV-LDI for various equivalence ratios $(\phi)$. As shown in the figure, the peak-to-peak pressure fluctuations are small and less than $0.10 \%$ of the inlet pressure. The highest pressure fluctuation measured was $2.14 \mathrm{kPa}$ at around $58.6 \mathrm{~Hz}$ at the maximum inlet conditions of $828 \mathrm{~K}, 2,413 \mathrm{kPa}$ and a $\phi=0.46$ as shown in Fig. 4. The ordinate scale in Fig. 3 has been reduced to better show the dynamic behavior at the higher frequencies. The other observation that can be made is the multiple peaks that seem to have some periodicity.

Figure 3 shows that the dynamic peak-to-peak pressure amplitude increases with increasing equivalence ratios. The same equivalence ratio behavior was observed at inlet pressures of 1,724 and $1,034 \mathrm{kPa}$ at inlet temperatures of 828 and $728 \mathrm{~K}$. This same behavior was also observed by DeLaat et al. ${ }^{4}$ They identified some of the same factors that contribute to instability growth as reference 15, like the phase relationship between the heat addition and the combustion chamber acoustics (Rayleigh criterion ${ }^{16}$ ), vortex shedding, other turbulent phenomena generated by the fuel injectors, and the energy introduced into the combustor by burning. For each of the plots in Fig. 3 and 4, the airflow, inlet temperature and pressure were held relatively constant while the equivalence ratio was increased by increasing the fuel flow. 
The dynamic behavior of this injector and flame tube configuration for the inlet conditions in Fig. 3 shows that the pressure spectrum is nominally the same for the equivalence ratios investigated and the only difference is the magnitude of the peak-to-peak fluctuations. The peaks do not shift with equivalence ratio as would be expected from classical acoustic wave theory. Depending on the system, the fundamental frequency, $f_{0}$, is given by the following expression, $f_{0}=\mathrm{c} 2 L$, for a closed system, where $\mathrm{c}$ is the speed of sound, which is a function of the gas temperature, and $L$ is the flame tube length. The gas temperature decreases with decreasing equivalence ratio and therefore the speed of sound will decrease accordingly since the flame tube length, $L$, is constant. The frequency should decrease

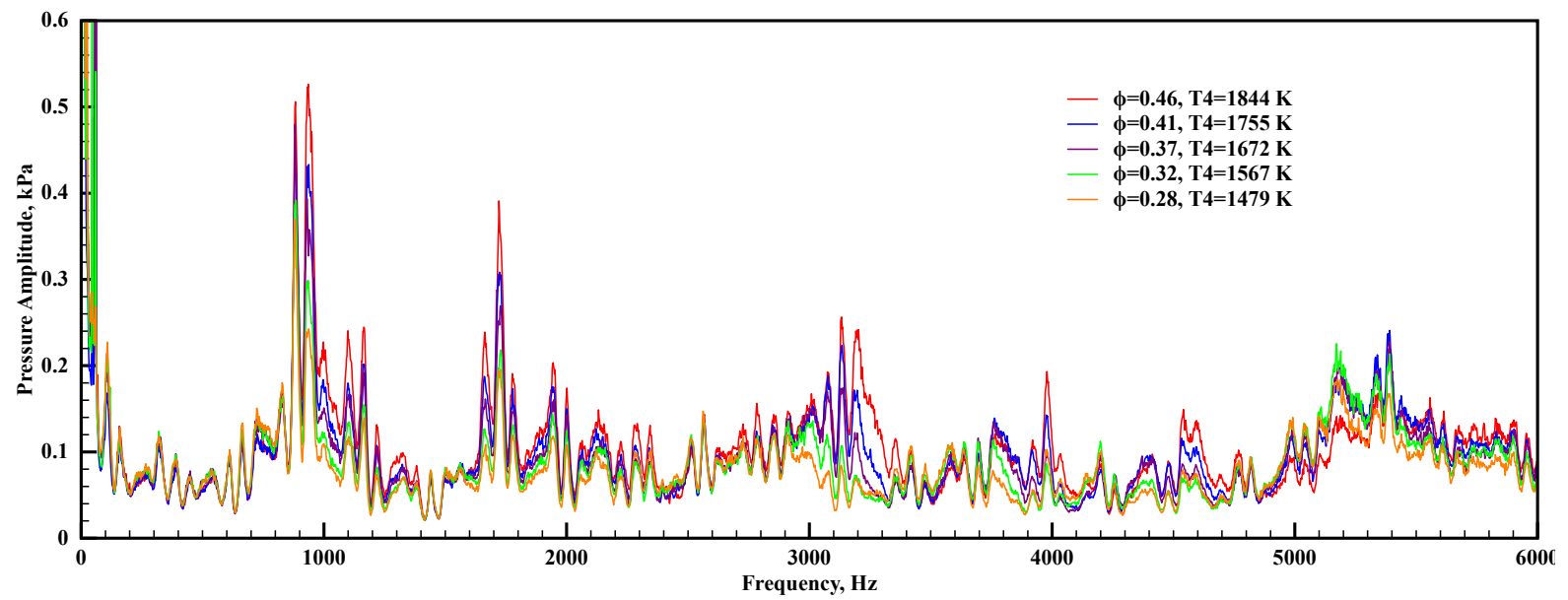

Figure 3. Pressure spectrum downstream of the SV-LDI at an inlet temperature of $828 \mathrm{~K}$ and inlet pressure of $2,413 \mathrm{kPa}$.

and it should be manifested as a shift of the peak-to-peak pressure spectrum to the left by as much as $80 \mathrm{~Hz}$ from the lowest equivalence ratio to the highest, but is not shown in Figs. 3 and 4.

We looked at the pressure spectrum during nonreacting and reacting conditions for inlet conditions of $728 \mathrm{~K}$ and $1724 \mathrm{kPa}$. As shown in Fig. 5, the spectra are very similar up to about $3000 \mathrm{~Hz}$. This and the fact that the pressure spectra does not scale with the speed of sound would point to the conclusion that only hydrodynamic modes are responsible for the dynamic characteristics of this injector and flametube combination at the conditions shown.

Phase Doppler interferometry (PDI) and planar particle image velocimetry (PIV) were used to measure the velocity fields of the nine point SV-LDI at the inlet temperature of $828 \mathrm{~K}, 728 \mathrm{~K}$, and $672 \mathrm{~K}$; and inlet pressure $1034 \mathrm{kPa}$ and $1379 \mathrm{kPa}^{19,20}$. It was found that the highest axial velocities occur at or near where the flows from the upper and lower injectors mix with that of the center injector and local turbulence values were maximum where adjacent fuel jets mixed. Measurements taken about $3 \mathrm{~mm}$ from the injector exit plane showed rotation about the injector centerline, which might indicate the presence of a

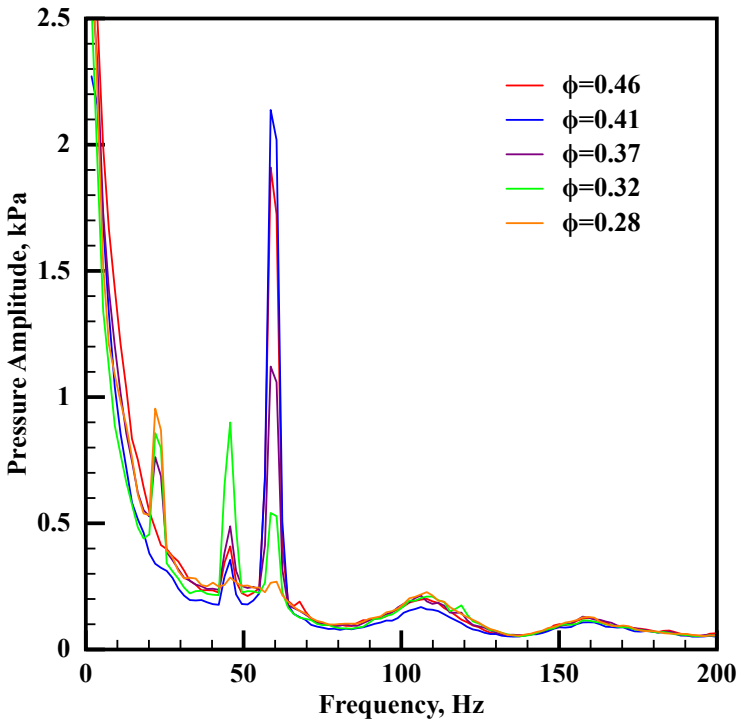

Figure 4. Low frequency pressure spectrum downstream of the SV-LDI at an inlet temperature of 827.6 K and inlet pressure of $2,413.2 \mathrm{kPa}$. 
An investigation of the swirling flow of the nine point SV-LDI under non-reacting atmospheric conditions found that at a distance of $30 \mathrm{~mm}$ downstream all the swirling jets merge together to form one large weak swirling flow keeping the original direction of rotation ${ }^{21}$. This may be an indication of a PVC along the length of the flame tube. Further analysis will be required to confirm if the PVC is still present and its size during reacting conditions.

As previously mentioned, Huang et al. ${ }^{7}$ utilized DMD analysis to help them get an insight into the dynamics of

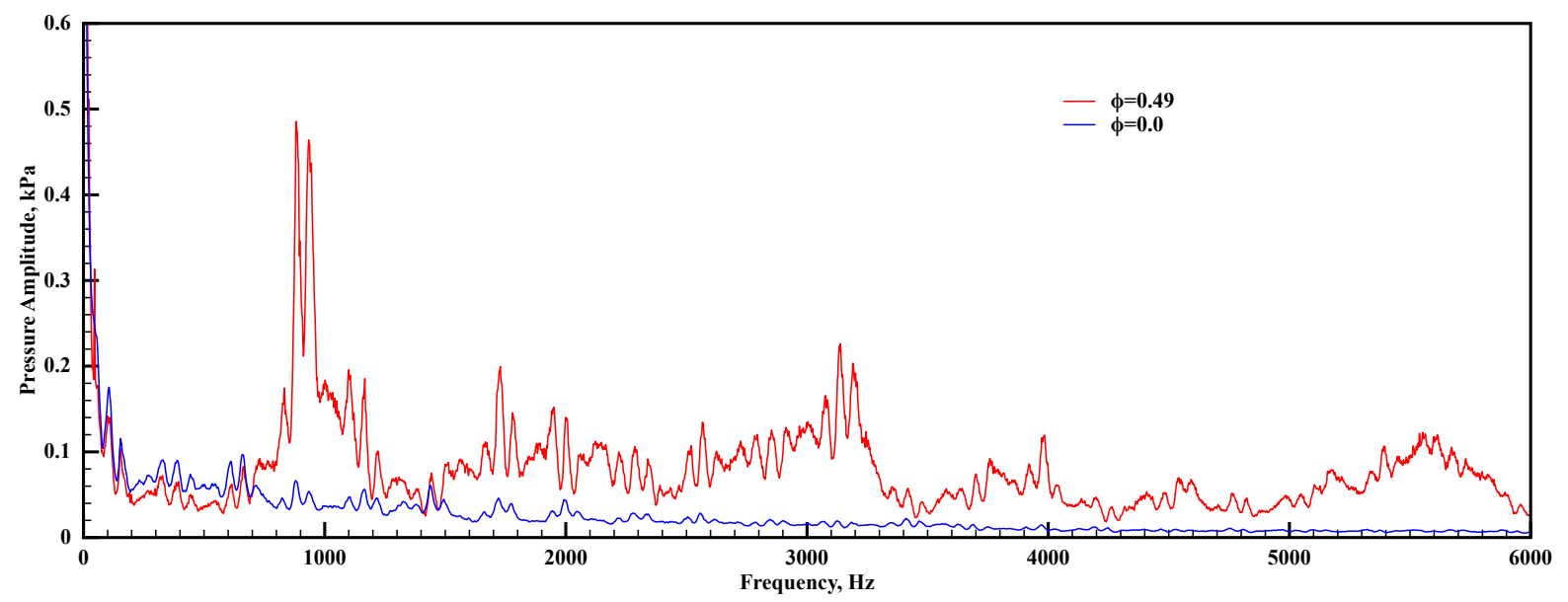

Figure 5. Relationship between non-reacting and reacting pressure spectrum at an inlet temperature of 727.6 K and inlet pressure of $1723.6 \mathrm{kPa}$.

their LDI combustor. Their analysis identified a coupling between axial acoustics and the vortex breakdown bubble (VBB) at lower frequencies. The PVC was found to be responsible for the dominant peaks at the higher frequencies between 6,000 and 8,000 Hz. Further analysis will be required to determine what role the VBB and the PVC play in the combustion dynamics of the nine-point SV-LDI flametube combustor.

The effect of inlet temperature and pressure was also studied. Figure 6 shows the effect of the inlet pressure on peak-to-peak dynamic pressure amplitude. The peak-to-peak pressure fluctuations increase with an increase in inlet pressure for constant inlet airflow and temperature. Lefebvre ${ }^{17}$, when discussing the influence of combustor operating conditions on combustion instabilities and the relationship between the combustion time scales and the acoustics, suggested that an increase in pressure would enhance the chemical reaction rates and reduce acoustic oscillations. Since combustion instabilities could be considered as a balance between the flame dynamics and acoustics coupling, the increase in combustion energy seems to be offsetting the acoustics thus sustaining the oscillations and resulting in an increase in dynamic pressure amplitudes. This effect could also be explained by plane wave theory. Lieuwen ${ }^{18}$ showed that under steady state conditions, constant time-averaged acoustic power flow and no reflections, the pressure and velocity are approximately related by the expression $p_{1}^{\prime}=\rho_{0} c_{0} u_{\mathrm{x}, 1}^{\prime}$. This expression shows that any changes in the mean properties, such as density increase due to an increase in pressure, will cause corresponding changes in the dynamic pressure and velocity. For the configuration studied here, this expression seems to indicate that the velocity fluctuations are less pronounced than the pressure fluctuations as indicated by the increase in the peak-to-peak pressure fluctuations. 


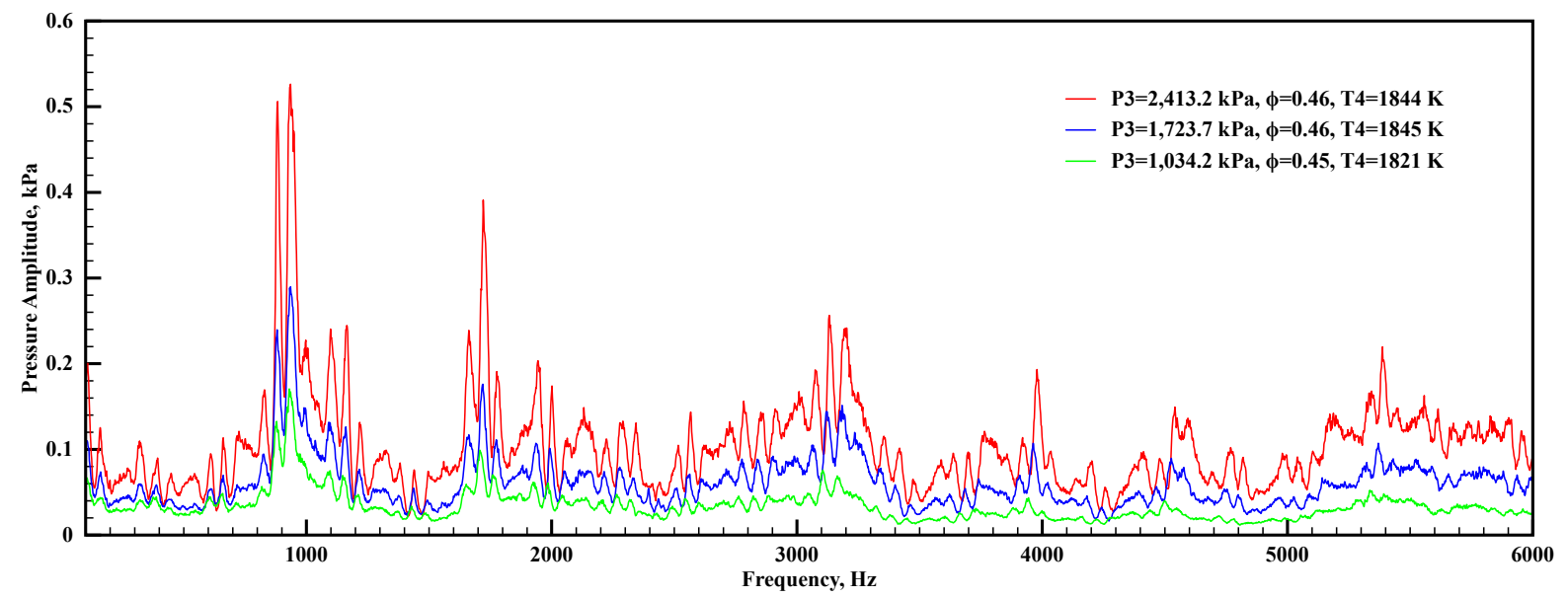

Figure 6. Pressure spectrum downstream of the SV-LDI at an inlet temperature of $828 \mathrm{~K}$ showing effect of inlet pressure on the combustion dynamics.

The effect of inlet temperature on the combustion dynamics is shown in Fig. 7. As discussed above in relation to inlet pressure, any factor that enhances the chemical reaction rates will tend to reduce instabilities ${ }^{15}$. PDI measurements of the droplet sizes for the SV-LDI showed that for a fixed inlet pressure, but different inlet temperatures, the higher inlet temperatures presented smaller fuel drops on average and that those drops vaporized more rapidly ${ }^{19}$. Increasing the inlet temperature reduces the evaporation time and the reaction time. Thus it should be expected that the peak-to-peak pressure fluctuations for the higher inlet temperature case will be lower. Figure 7 does show some of that behavior. The peak-to-peak pressure fluctuations for the $828 \mathrm{~K}$ inlet temperature case (blue line) are in general lower than the lower inlet temperature of $728 \mathrm{~K}$ (red line).

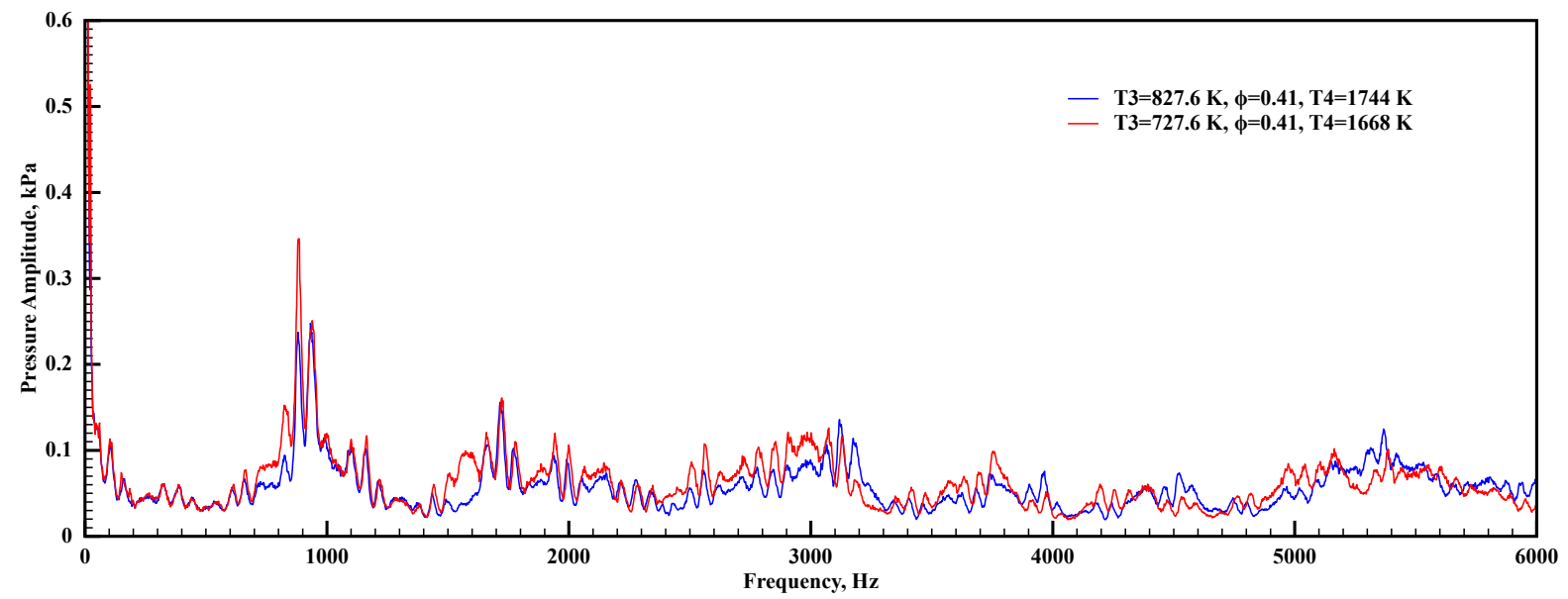

Figure 7. Pressure spectrum downstream of the SV-LDI at an inlet pressure of $1,724 \mathrm{kPa}$ showing the effect of inlet temperature on combustion dynamics.

\section{B. Fuel Staging}

All the data presented up to this point has been with all nine fuel injectors flowing the same amount of fuel to represent a mid power to high power condition. For low power and idle conditions it may be necessary to shift fuel to a particular injector or set of injectors for low power combustion stability purposes. During this experimental campaign we decided to investigate the combustion dynamics when the pilot injector, the center one in Fig. 2, was richer than the other eight. The results are shown in Fig. 8. The figure on the left shows two very large peaks for the two conditions shown. The spectra were taken at the same inlet temperature, pressure, and mass flow conditions. Changing the fuel flow varied the equivalence ratio. Two large peaks are observed at 47.6 and $95.2 \mathrm{~Hz}$ that are much larger in magnitude than the others.

American Institute of Aeronautics and Astronautics 

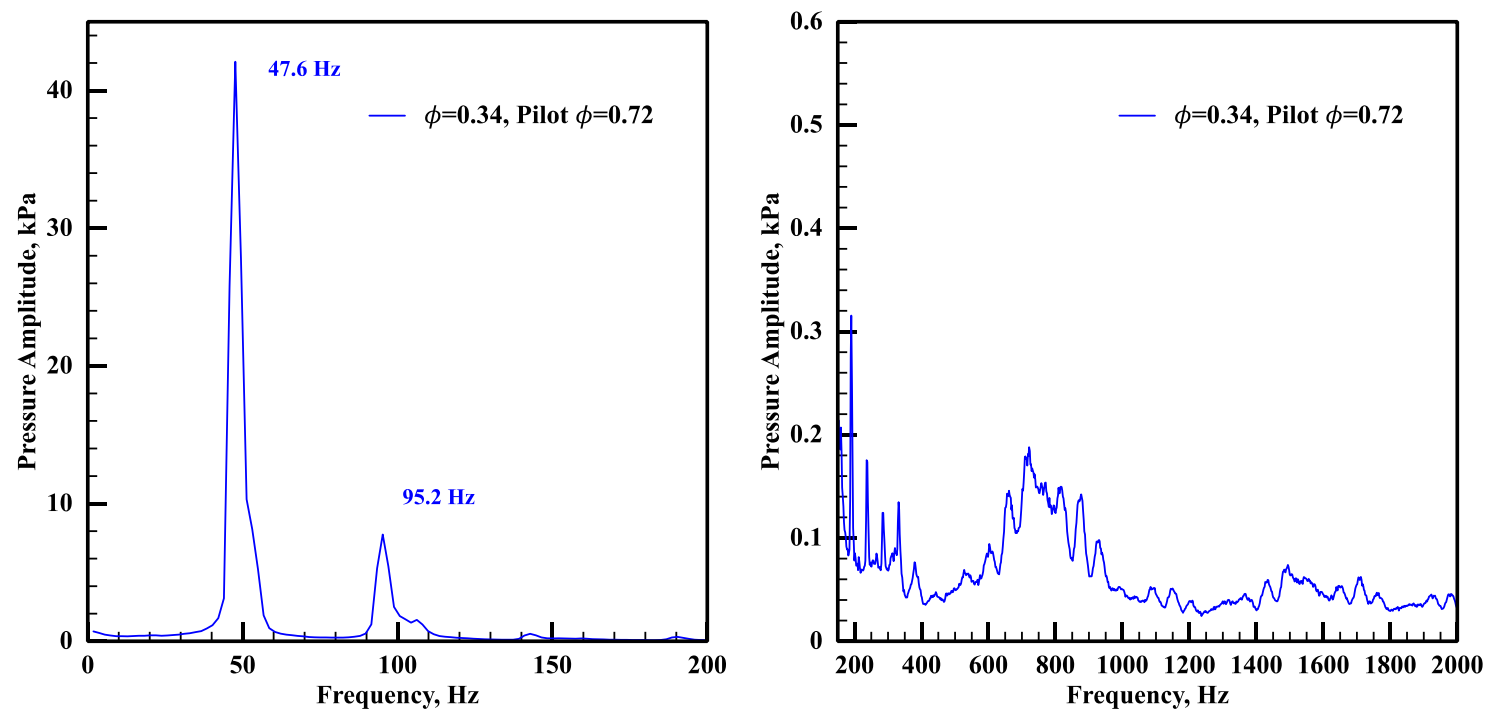

Figure 8. Pressure spectrum downstream of the SV-LDI at an inlet pressure of $1,117 \mathrm{kPa}$ and inlet temperature of $653 \mathrm{~K}$ showing the effect of fuel shifting on combustion dynamics.

Low-frequency instabilities have been referred to by difference names i.e., "lean-limit", "cold tone", an "incipient blowout", "entropy wave" or "equivalence ratio oscillation.", 9, 15 . Dhanuka et al. ${ }^{8}$ studied a lean-limit instability and identified physical mechanism causing the instability. Utilizing high-speed movies they observed that as the flame enters the shear layer, is locally extinguished and lifts up, the extinguished region fills with unburned reactants again, the flame flashbacks igniting the unburned reactants creating pressure rise and starting next cycle.

An equivalence ration oscillation was studied by Temme et al. ${ }^{9}$ in a lean premixed prevaporized gas turbine flametube. Utilizing high-speed images and pressure measurements they found that the airflow rates were oscillating and concluded that the "equivalence ratio oscillation" couples with a Hemholtz tone.

The entropy wave mode has been described as a vortex generated in the flame region and convected toward the nozzle impinging on it and exciting an acoustic wave that propagates back to the flame. This process excites another convected wave repeating the process again ${ }^{15}$.

What we observed and is shown in Fig. 8 could be any of the modes mentioned or even a combination of those modes. Eight of the nine fuel injectors were operated at very lean condition, $\phi=0.26$, while the pilot's equivalence ratio was 0.71 for a global equivalence ratio of 0.31 . Further analysis is needed to pinpoint the most likely mode present in our flametube combustor.

\section{Summary and Conclusions}

The combustion dynamic characteristics of a research nine-point swirl venturi LDI flametube under simulated gas turbine combustor conditions were experimentally investigated. The SV-LDI geometry was evaluated at inlet pressures up to 2,413 $\mathrm{kPa}$ and non-vitiated air temperatures up to $867 \mathrm{~K}$. When the SV-LDI was operated with all the injectors flowing the same amount of fuel, i.e., same local equivalence ratio, the dynamic characteristics were found to be relatively small, less than $0.10 \%$ of the inlet pressure. The peaks were not acoustically coupled and most likely driven by hydrodynamic modes. The swirl venturi injector is responsible for the peaks in the frequency spectrum for frequencies up to about $3000 \mathrm{~Hz}$. This was confirmed by comparing the non-reacting frequency spectrum to the reacting one. The instabilities above $3000 \mathrm{~Hz}$ present during combustion may indicate the presence of a PVC. Those get amplified by the combustion process. Peak-to-peak dynamic pressure fluctuations larger than $40 \mathrm{kPa}$ were measured at $653 \mathrm{~K}$ inlet temperature and $1117 \mathrm{kPa}$ inlet pressure when the pilot fuel injector equivalence ratio was increased to 0.71 and the other fuel injectors' equivalence ratio was reduced to 0.26 . A leanlimit instability, equivalence ratio oscillation, or entropy wave or a combination of those is a possible operating mode.

\section{Acknowledgments}

This work was supported by the Advanced Air Transport Technology Program. 


\section{References}

${ }^{1}$ Tacina, R., Lee, P., and Wey, C., “A Lean-Direct-Injection Combustor Using a 9 Point Swirl- Venturi Fuel Injector,” 17th International Symposium of Airbreathing Engines (ISABE), ISABE-2005-1106, Munich, Germany, 2005.

${ }^{2}$ Lilly, D. G., "Swirling Flow in Combustion: a Review," AIAA J., Vol. 15, No. 8, 1977, pp. 1063-1078

${ }^{3}$ Candel, S., Durox, D., Schuller, T., Brourgouin, J.-F., and Mocek, J. P., "Dynamics of Swirling Flames," Annual Review of Fluid Mechanics, Vol. 46, 2014, pp. 147-173.

${ }^{4}$ DeLaat, J.C., Paxzon, D.E, "Characterization and Simulation of the Thermoacoustic Behavior of an Advanced, Low Emissions Combustor Prototype," NASA TM-2008-215291, AIAA-2008-4878

${ }^{5}$ Yi, T. and Domenic, S., "Flame Spectra of a Turbulent Liquid-Fueled Swirl-Stabilized LDI Combustor," in 47th AIAA Aerospace Sciences Meeting including The New Horizons Forum and Aerospace Exposition. 2009, American Institute of Aeronautics and Astronautics.

${ }^{6} \mathrm{Yi}, \mathrm{T}$. and Domenic, S., Combustion Instability in a Turbulent Liquid-Fueled Swirl-Stabilized LDI Combustor, in 45th AIAA/ASME/SAE/ASEE Joint Propulsion Conference \& Exhibit. 2009, American Institute of Aeronautics and Astronautics.

${ }^{7}$ Huang, C., Gejji, R., and Anderson, W. E., "Combustion Dynamics Behavior in a Single-Element Lean Direct Injection (LDI) Gas Turbine Combustor,” AIAA 2014-3433, 2014.

${ }^{8}$ Dhanuka, S.K., Temme, J.E., Driscoll, J.F., "Lean-limit combustion instabilities of a lean premixed prevaporized gas turbine combustor" Proc. Combust. Inst., Vol. 33, 2011, pp. 2961-2966.

${ }^{9}$ Temme, J.E., Allison, P.M., Driscoll, J.F., "Combustion instability of a lean premixed prevaporized gas turbine combustor studied using phase-averaged PIV," Combustion and Flame 161 (2014) 958-970.

${ }^{10}$ Tacina, K. M., Chang, C.T., Lee, P., Mongia, H., Podboy, D. P., and Dam, B., “Assessment of Combustion Dynamics in a Second-Generation Swirl-Venturi Lean Direct Injection Combustion Concept," ISABE-2015-20249

${ }^{11}$ Szedlmayer, M. T., "An Experimental Study of the Velocity-Forced Flame Response of a Lean-Premixed Multi-Nozzle Can Combustor for Gas Turbines,” Ph. D. Thesis, The University of Pennsylvania, August 2013.

${ }^{12}$ Beer and N.A. Chigier. Combustion Aerodynamics. John Wiley \& Sons, Inc., New York, 1st edition, 1971.

${ }^{13}$ Englund, D. R. and Richards, W. B, "The Infinite Line Pressure Probe,” NASA TM-83582, 1984.

${ }^{14}$ Samuelson, R. D., "Pneumatic Instrumentation Lines and Their Use in Measuring Rocket Nozzle Pressure," Report No. RN-DR-0124, Nuclear Rocket Operations, Aerojet-General Corporation, 1967.

${ }^{15}$ B.T. Zinn, T.C. Lieuwen, in: T.C. Lieuwen, V. Yang (Eds.), AIAA Progress in Astronautics and Aeronautics, vol. 210, 2005, pp. 3-26 (Chapter 1).

${ }^{16}$ Rayleigh, J.W.S., "The Theory of Sound," Vol. 2, 1896, Macmillan and Co.

${ }^{17}$ Lefebvre, A. H., Gas Turbine Combustion, Taylor and Francis, Philadelphia, 2nd ed., 1998.

${ }^{18}$ Lieuwen, T. C., Unsteady Combustor Physics, Cambridge University Press, Cambridge, 1st ed., 2013

${ }^{19}$ Heath, C.M, Anderson, R.C., Locke, R.J., Hicks, Y.R., "Optical Characterization of a Multipoint Lean Direct Injector for Gas Turbine Combustors: Velocity and Fuel Drop Size Measurements,” IGTI, GT2010-22960, 2010.

${ }^{20}$ Hicks, Y.R., Heath, C.M., Anderson, R.C., and Tacina, K.M., "Investigations of a Combustor Using a 9-Point SwirlVenturi Fuel Injector: Recent Experimental Results" ISABE-2011-1106

${ }^{21} \mathrm{Fu}$, Y., Jeng, S-M, "Characteristics of the Swirling Flow in a Multipoint LDI Combustor," in 47th AIAA Aerospace Sciences Meeting, 8-11 January 2007, Reno, NV, AIAA 2007-846 